\title{
Thermo Acoustical Studies of Solar Nanophotocatalytic Degradation of Alizarin Red S by using CuO Nanophotocatalyst
}

\author{
Nita P. Mohabansi*, Anita K. Satone \\ Department of Chemistry, Jankidevi Bajaj College of Science, Wardha - 442 001, Maharashtra, India.
}

\section{ARTICLE DETAILS}

Article history:

Received 20 February 2018

Accepted 28 February 2018

Available online 02 March 2018

\section{Keywords:}

Nanophotocatalytic

Thermoacoustical Parameters

Intermolecular Interactions

\begin{abstract}
A B S T R A C T
Anthraquinone dyes represent the largest class of textile dyes in industrial use. These reactive anthraquinone dyes are not easily amenable by conventional treatment methods due to their stability and non-biodegradable nature. Heterogeneous solar nano photocatalysis has been found to be the most promising treatment process for the degradation of various dyes. In the present work alizarin red $S$ is used as model compound for the nanophotocatalytic degradation. The effects of operational variables such as catalyst dose, $\mathrm{pH}, \mathrm{H}_{2} \mathrm{O}_{2} / \mathrm{COD}$ ratio are investigated. The complete color removal is achieved at optimum conditions of catalyst dose of $7 \mathrm{mg} / 500 \mathrm{~mL}, \mathrm{pH}=7$ and contact time $95 \mathrm{~min}$ for dye sample. This is the first attempt made to analyse the degradation pathway of the dye by the thermo acoustical studies of solar nanophotocatalytic degradation of alizarin red S. Based on the data obtained, various acoustical parameters like adiabatic compressibility, intermolecular free length, acoustic impedance, relative association, solvation number, vander Waal's constant, Rao's constant and relative association have been calculated. From the data of acoustical parameters, the intermolecular interactions between the selected dye, $\mathrm{H}_{2} \mathrm{O}_{2}$ and the nano photocatalyst have been discussed and the mechanism of nanophotocatalytic degradation of alizarin red $\mathrm{S}$ is proposed.
\end{abstract}

\section{Introduction}

A textile industry is one of the oldest and largest industries in the world. It produces large quantities of highly coloured effluents, which are generally toxic and carcinogenic nature [1-6]. Continually release of wastewater containing dyes into water bodies is growing a global pollution problem as a result of their wide industrial uses. In aquatic environment, the dyes undergo biochemical changes and consume dissolved oxygen, which further threat to the living organism in aquatic ecosystem as well as human health [7-9]. Consequently, it is definitely necessary to remove the dye contaminant before discharging them into streams [10-12]. The various conventional technologies such as precipitation, adsorption, air stripping, flocculation, reverse osmosis a textile effluents. These currently employed methods for the removal of coloured effluents in industrial water are classical and do not lead to complete destruction of the dyes. These methods do not work efficiently due to high solubility of dyes as well as their resistance to chemical and biological degradation. These techniques are considered to be nondestructive, since they only transfer the non-biodegradable matter into sludge, giving rise to new type of pollution, which needs further treatment [13-18].

Recently there has been considerable interest in the utilization of advanced oxidation processes (AOPs) for the complete destruction of dyes. AOPs are based on generation of reactive species such as hydroxyl radicals that oxidizes a broad range of organic pollutants quickly and nonselectively [19-23]. Nano photo catalytic AOPs include combination of semiconductors, light and oxidants. Heterogeneous nanophoto catalysis has emerged as an important destructive technology leading to the total mineralization of most of the organic pollutants including organic reactive dyes [24-26]. In the tropical countries use of solar irradiation is very attractive technology from the economical point of view.

In the recent years, ultrasonic technique has become a more powerful tool in providing information regarding the behaviour of liquids and solids owing to its ability of characterizing physiochemical behaviour of the medium [27-30]. An ultrasonic study gives the information about the nature of molecular interaction in the solute-solvent and solvent-solvent

mixture and it plays an important role in the development of molecular sciences. These interaction help in better understanding the nature of solute and solvent i.e. whether the solute modifies or distorts the structure of the solvent. It is non-destructive and very effective technique for the investigation of various thermo dynamic parameters [31]. It is revealed from the literature survey that solar nano photocalytic degradation of alizarin red $\mathrm{S}$ and its correlation with the thermo acoustical parameters have not been reported so far.

The aim of the present work is to optimize the factors affecting solar nano photocatalytic degradation of substrate alizarin red $\mathrm{S}$ viz. amount of nanocatalyst dose, $\mathrm{H}_{2} \mathrm{O}_{2} / \mathrm{COD}$ ratio, $\mathrm{pH}$. This is the first attempt made to analyse the degradation pathway of the dye by the thermo acoustical studies of solar nanophotocatalytic degradation of alizarin red S. At an optimised conditions nanophotocatalytic AOPs, acoustical parameters like adiabatic compressibility, intermolecular free length, acoustic impedance, relative association, solvation number, Vander Waal's constant, Rao's constant and relative association were determined. This would help to understand the solute and solvent interaction and to propose the mechanism of degradation of alizarin red S.

\section{Experimental Methods}

\subsection{Materials}

All AR grade chemicals were used in the experiments. Also, these chemicals used as received without further purification. The solutions were prepared in double distilled water. All stock solutions were stored in amber coloured light resistant pyrex glass bottles. Sodium hydroxide (1 $\mathrm{M})$ and sulphuric acid (1 M) were used for $\mathrm{pH}$ adjustment.<smiles>O=C1c2ccccc2C(=O)c2c1cc(S(=O)(=O)O)c(O)c2O</smiles>

Fig. 1 Structure of alizarin red S (Molecular Formula: $\mathrm{C}_{14} \mathrm{H}_{7} \mathrm{NaO}_{7} \mathrm{~S}$; Average mass: 342.25589; C.I. No. 58005; Scientific name -Sodium3,4-dihydroxy-9,10-dioxo9,10-dihydro-2- anthracenesulfonate) 


\subsection{Experimental Procedures}

In the experiment a stock solution of the substrate of conc. $1 \times 10^{-2} \mathrm{~mol} / \mathrm{L}$ was used. Batch experiments were carried out to determine the effect of $\mathrm{pH}, \mathrm{H}_{2} \mathrm{O}_{2}$ concentration, and nanophotocatalyst $\mathrm{CuO}$ doses during degradation of selected dye. The solar photocatalytic degradation was carried out in $1 \mathrm{~L}$ flat glass vessel provided with an aquarium pump for the continuous agitation of the sample. During the reaction, the solution was stirred by magnetic pellet to ensure its homogeneity. $500 \mathrm{~mL}$ of the substrate was taken in the photo reactor and irradiated with sunlight. Samples were pipetted at regular time interval from the reaction vessel. Then a centrifugation process was applied to separate $\mathrm{CuO}$ catalyst particles after photo catalysis. The samples were immediately analysed to avoid further reaction.

\subsection{Analysis}

The $\mathrm{pH}$ of the solution was measured using Elico pH meter LI-120 equipped with a combined calomel-glass electrode. Shimadzu UV-Visible 1800 Spectrophotometer was used for the measurement of absorbance of the substrate. A calibration plot between absorbance and concentration of dye was plotted experimentally, which gave a high linear regression coefficient of 0.987 at $430 \mathrm{~nm}$ (Fig. 2).

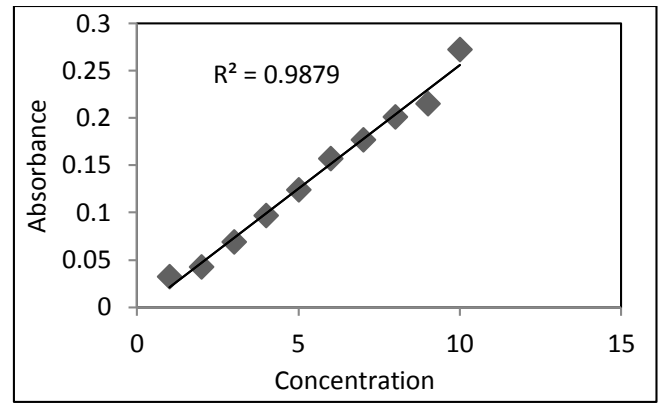

Fig. 2 Calibration plot of Alizarin Red S

The progress of the photocatalytic degradation of the dye was monitored by taking out aliquots at regular intervals and recording absorbance by UV-VIS spectrophotometer. The percentage of degradation was calculated by the formula

$$
\text { Percentage degradation }=\frac{(\mathrm{Co}-\mathrm{C})}{\mathrm{Co}} \times 100
$$

where $\mathrm{Co}=$ initial substrate concentration

$\mathrm{C}=$ final substrate concentration at a given time.

\subsection{Thermoacostical Study of the Substrate at Optimized Parameters}

The densities $(\rho)$ of these binary solution were measured accurately using $25 \mathrm{~mL}$ specific gravity bottle in an electronic balance precisely and accurately. The basic parameter ultrasonic velocity $(v)$ has been measured on Digital Ultrasonic Pulse Echo Velocity Meter (Vi Microsystems Pvt. Ltd. Model VCT-70 at $2 \mathrm{MHz}$ ) with an accuracy of $0.1 \%$. By using ultrasonic velocity and density the following acoustical parameters like adiabatic compressibility, intermolecular free length, acoustic impedance, relative association, solvation number, Vander Waal's constant, Rao's constant and relative association were calculated by applying the following expressions [32].

$$
\begin{aligned}
& \text { Ultrasonic velocity } \quad U=f \cdot \lambda \\
& \text { Adiabatic compressibility: }
\end{aligned}
$$

$(\mathrm{U}=$ Ultrasonic velocity of solution, $\rho=$ Density of the solution $)$

$$
\begin{aligned}
& \text { Acoustic impedance } \mathrm{Z}=\mathrm{U} \times \rho \\
& \text { Intermolecular free length }{ }^{L_{f}}=\mathrm{K}_{T} \times \sqrt{\beta} \\
& \text { (KT is Jacobson's constant, }{ }^{\mathrm{T}}=(93.875+0.375 \mathrm{~T}) \times 10^{-8} \text { and } \\
& \mathrm{T}=\text { Absolute temperature in Kelvin) }
\end{aligned}
$$

$$
\begin{aligned}
& \text { Rao's constant } \mathrm{R}=\left(\frac{M}{\rho}\right) v_{2} \\
& \mathrm{M}=\text { Mol. Wt. }
\end{aligned}
$$

Wada's constant $\mathrm{W}=\mathrm{M} \mathrm{K}^{-1 / 7} / \rho$

Relative association $=\frac{\rho}{\rho 0}$

$\rho$ and $\rho 0$ are the densities of the solution at time $t$ and $t=0$ resp.

Solvation Number $=\mathrm{M} / \mathrm{M} 0\left(1-\frac{\beta}{\beta 0}\right)\left(\frac{100-x}{x}\right)$
Vander Waal constant $=\frac{M}{\rho}\left[1-\frac{R T}{M V 2} \sqrt{1+\frac{M V 2}{3 R T}}-1\right]$

$\mathrm{M}=$ Mol. Wt., $\mathrm{V}$ is the volume of the solution

Relative Association $=\left(\rho / \rho^{0}\right)\left(U_{0} / U\right)^{1 / 3}$

where $\rho=$ Density of liquid mixture

$\rho^{0}=$ Density of pure liquid mixture

$\mathrm{U}=$ Ultrasonic velocity of solution

$\mathrm{U}_{0}=$ Ultrasonic velocity of solution

\section{Results and Discussion}

\subsection{Effect of $p H$}

$\mathrm{pH}$ is one of the most important factor for the generation of hydroxyl radicals. An attempt has been made to study the effect of $\mathrm{pH}$ on the degradation of substrate under sunlight. It has been observed that at optimal concentration of dyes in both acidic and alkaline $\mathrm{pH}$ tends to lower the degradation efficiency of anthraquinone dye. The maximum degradation was observed at $\mathrm{pH} 7$ as shown in (Fig. 3). This is may be due to the fact that at high $\mathrm{pH}$ values the hydroxyl radicals are so quickly discarded that they do not have proper time to react with dyes in waste water. Also the $\mathrm{pH}$ influences the surface properties of $\mathrm{CuO}$ nanophotocatalyst, dissociation of organic pollutants (or dyes in waste water) and formation of hydroxyl radicals [25]. Therefore, the result indicates that $\mathrm{pH}$ value has a significant effect on the adsorption properties at the photocatalyst surface and hence photodegradation rates are quite insignificant with extreme $\mathrm{pH}$ values. Photo degradation is optimized at pH 7.

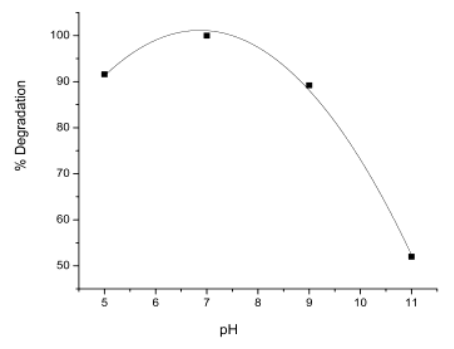

Fig. 3 Effect of pH on the degradation

\subsection{Effect Nano CuO Catalyst}

Fig. 4 shows the effect of nanophotocatalyst loading on the rate of degradation of the selected substrate at an optimized $\mathrm{pH}$ 7. The photo degradation was studied by varying catalyst dose from 3 to $15 \mathrm{mg} / 500 \mathrm{~mL}$. The percentage degradation of the substrate increases with an increase in the dosage of the photo catalyst. However with further increase in photocatalyst doses decreases the percentage degradation of the dye. The maximum degradation efficiency was attained with $7 \mathrm{mg}$ of $\mathrm{CuO}$ nanophotocatalyst and thereafter the rate of degradation decreased. The increase in degradation rate may be explained by the fragmentation of catalyst which produces higher surface area. Thereafter with further increase in catalyst loading, the degradation rate starts declining. This nature is due to the screening effect i.e., above a certain amount of catalyst loading, the turbidity of the solution increases and ultraviolet rays start getting scattered, hence reducing the optical path $[33,34]$. The aggregation of catalyst particles, which reduces the interfacial area between the substrate solution and the nano photocatalysts may be other reason for the decrease in the degradation rate [35].

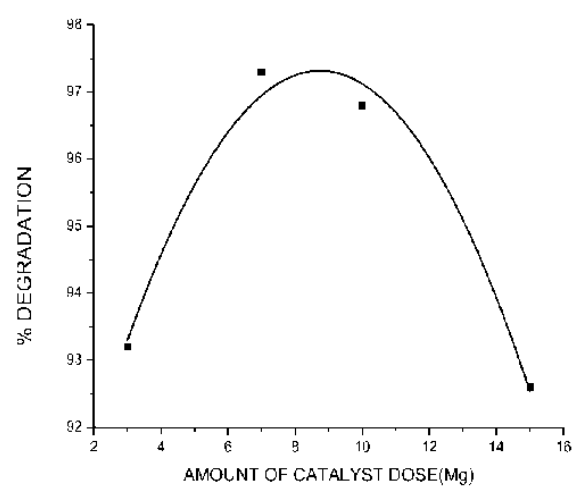

Fig. 4 Effect of nano photocatalyst doses 


\subsection{Effect of Dose of $\mathrm{H}_{2} \mathrm{O}_{2} / \mathrm{COD}$ Ratio Degradation of Selected Dye}

A series of experiments of substrate with different $\mathrm{H}_{2} \mathrm{O}_{2} / \mathrm{COD}$ ratios viz. $1,2,3 \& 4$ were carried out to optimize $\mathrm{H}_{2} \mathrm{O}_{2}$ /COD ratio. It was optimized at 1 (Fig. 5). Further increase in $\mathrm{H}_{2} \mathrm{O}_{2}$ concentration lowered the degradation rate. This is because of the excess $\mathrm{H}_{2} \mathrm{O}_{2}$ reacts with the hydroxyl radicals earlier formed and hence acts as an inhibiting agent of degradation by consuming the hydroxyl radicals responsible for degrading the substrate molecule.

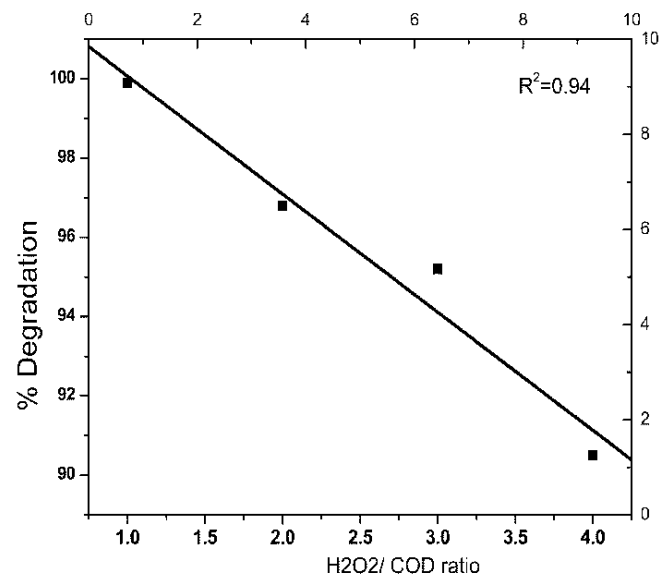

Fig. 5 Effect of $\mathrm{H}_{2} \mathrm{O}_{2} / \mathrm{COD}$ ratio on the degradation

The measured values of adiabatic compressibility, intermolecular free length, acoustic impedance, relative association, solvation number, Vander Waal's constant, Rao's constant and relative association of nanophotocatalytic degradation of the substrate at an optimized parameters viz. $\mathrm{pH}, \mathrm{H}_{2} \mathrm{O}_{2} / \mathrm{COD}$ ratio and nanophocatalyst dose with the exposure time are incorporated. The graphical representation of the data obtained from the measurement of the various parameters are given in Figs. 6-13.

At the optimized experimental condition the value of various parameters viz. adiabatic compressibility, intermolecular free length and intermolecular relative association increases with the exposure time. But the ultrasonic velocity, acoustic impedance, molar sound velocity (Rao's constant), Wada's constant, Vander Waal's constant and solvation number decreases nonlinearly.

It is seen that ultrasonic velocity decreases linearly with the exposure time. This descending value of ultrasonic velocity may be due to decrease in the no. of particles of solute. This indicates that with the increase in the exposure time, the rate of degradation of solute particles with the exposure time is also decreases \& hence ultrasonic velocity also decreases. Hence, there is no transfer of sound energy from one particle to another which is confirms the observation of the nano photocatalytic degradation study.

From Figs. 6 and 7, it is clearly shown that the values of adiabatic compressibility \& intermolecular free length increases linearly with the increase in the exposure time. The increase in the values of adiabatic compressibility and intermolecular free length with respect to increase in the exposure time indicates that the solute-solvent interaction decreases with the exposure time. As a result the structural arrangement is attended. This ascending value of adiabatic compressibility and intermolecular free length with respect to exposure time shows that there is breaking of bonds formed between the solute and solvent molecules [20, 21] and also observed in the degradation study.

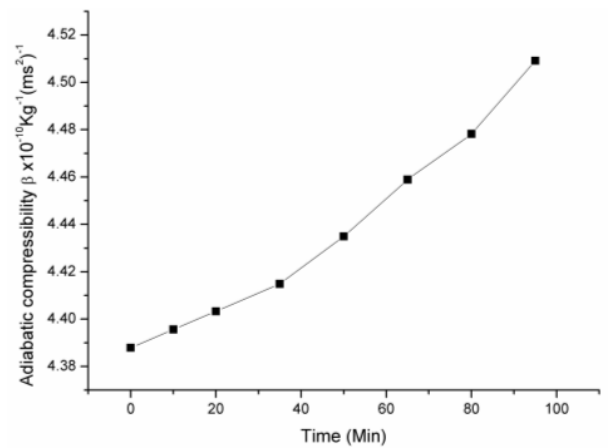

Fig. 6 Adiabatic compressibility vs Time

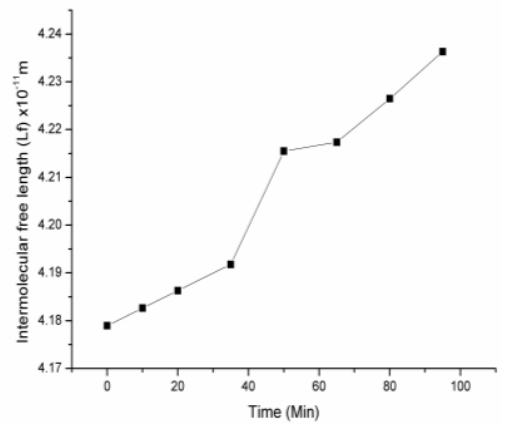

Fig. 7 Intermolecular free length vs Time

Similarly the values of acoustic impedance decreases with the exposure time while the relative association increases linearly. The ascending trend of the relative association indicates the increase in the strength of interactions between unlike molecules. This may be due to the hydrophilic nature of the interacting molecules \& the interaction decreases with the exposure time (Figs. 8 and 9).

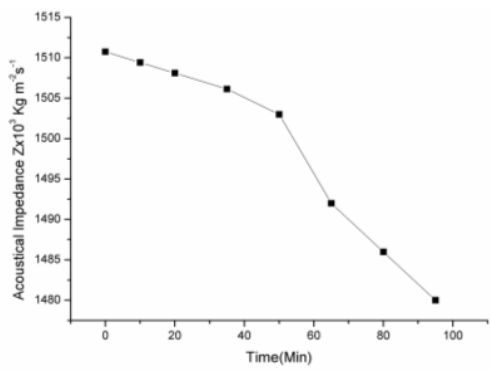

Fig. 8 Acoustic Impedance vs Time

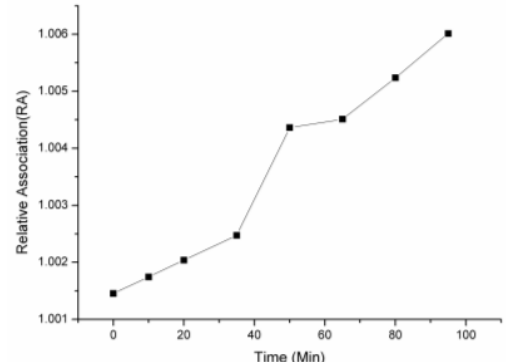

Fig. 9 Relative Association vs Time

The molar velocity (R) indicates the cube root of ultrasound velocity through 1 molar volume of the mixture and is also called as Rao's constant. It gives the interaction exist in the solution. The descending trend of the Rao's constant with the exposure time indicates the interaction decreases with the time (Fig. 10). While Wada's constant depends on the molar compressibility. It gives the confirmation for an existing interaction in the solution. In this study shows the decrease in the Wada's constant with the time Fig. 11.

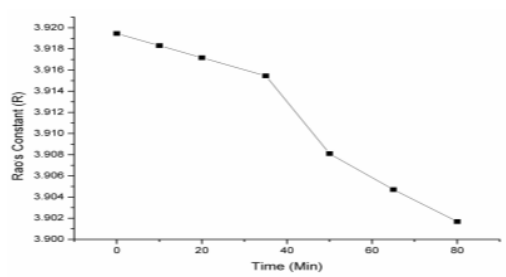

Fig. 10 Rao's Constant vs Time

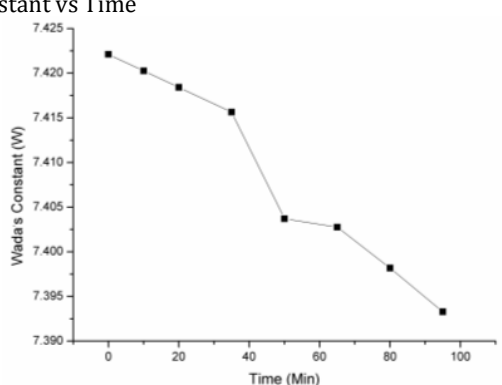

Fig. 11 Wada's constant vs Time 
This indicates that the interaction between solute and solvent is decreasing with the time. This may be due to insufficient amount of $\mathrm{H}_{2} \mathrm{O}_{2}$ after time $60 \mathrm{~min}$. Hence, the observed trends of Rao's constant and Wada's constant indicate that the magnitude of interactions is decreasing with the increasing time intervals.

The degree of solvation is the number of solvent molecules per ion, which remains attached to a given ion, long enough to experience its translation movement when solution is formed. Structure of the surrounding molecules also affects the solvation number. The compressibility of the solution is mainly due to the free solvent molecules. Fig. 12 shows the descending order of the solvation number with the exposure time. This may be due to fact that with the increase of the exposure time the solute and solvent interaction is decreasing. Thus the have more probability of contacting solvent molecules. Vander Waal's constant is an indicator of molecular volume. Fig. 13 shows the descending order of Vander Waal's constant with time. This indicates decrease in the molecular volume and thus the no. of solute molecules decreases with the exposure time and hence finally substrate is completely depredated.

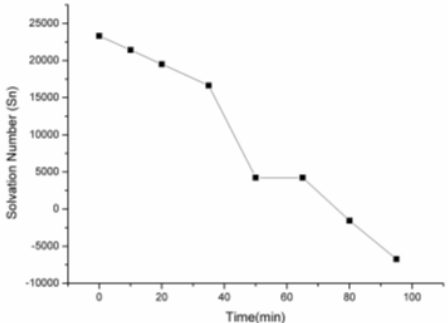

Fig. 12 Solvation Number vs Time

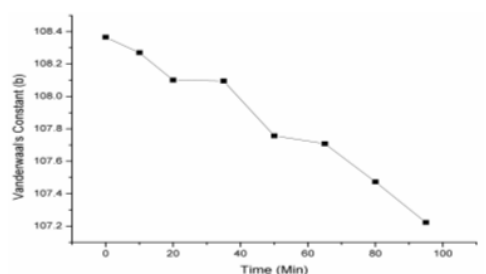

Fig. 13 vander Waal's Constant vs Time

On the basis of this study an attempt is made to propose the mechanism of the degradation of the alizarin red S as shown in Fig. 14.

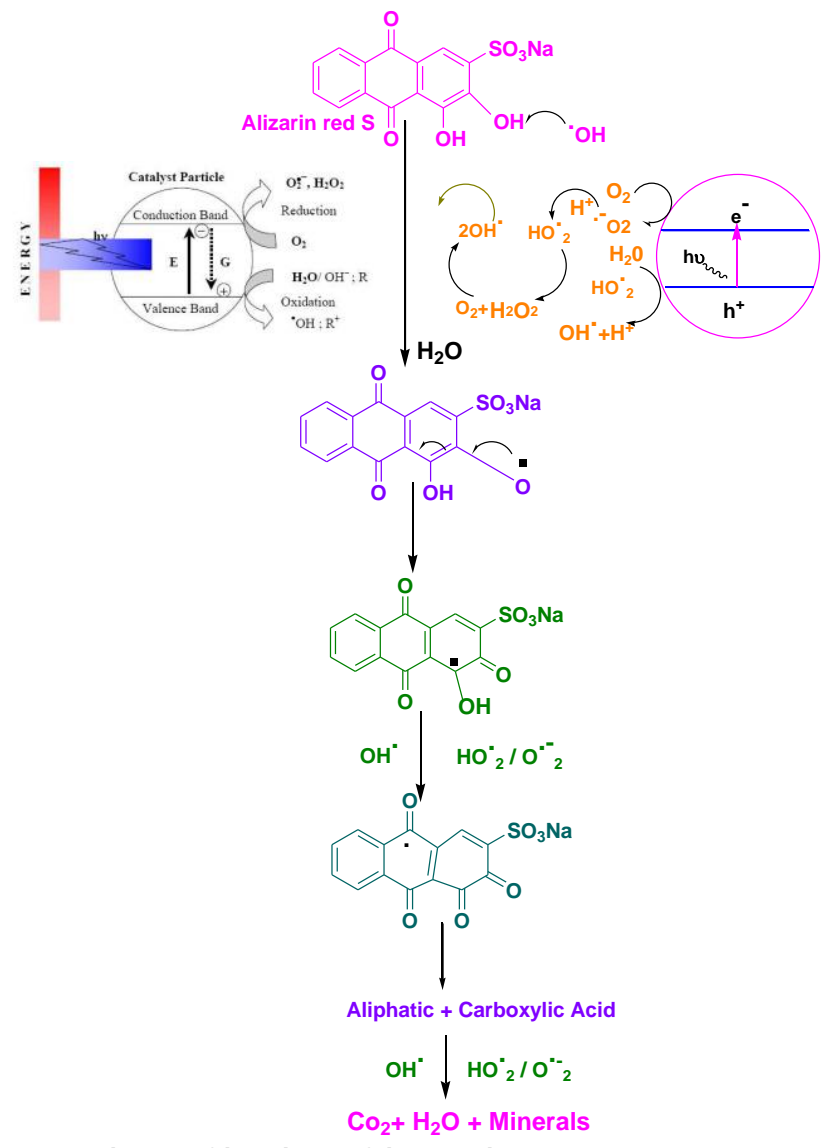

\section{Conclusion}

In the present study, the wastewater containing dye was degraded by using solar nano photocatalytic advanced oxidation process. The results obtained in the present study show the great efficiencies of advanced oxidation processes in removing anthraquinone dyes which are resistant to other conventional treatment processes. This is the first report on the thermo acoustical photocatalytic degradation of alizarin red $\mathrm{S}$. This analysis could be useful for suggesting the mechanism of degradation of the substrate. The application of heterogeneous photocatalytic treatment using $\mathrm{CuO}$ for the degradation of alizarin red $\mathrm{S}$ has been found to be promising process. Further addition of $\mathrm{H}_{2} \mathrm{O}_{2}$ to the above system made the degradation much faster and could degrade the dye. The observations of these investigations clearly demonstrated the importance of choosing the optimum degradation parameters which are essential for any practical application of photocatalytic oxidation process. This technique is ecofriendly because it uses conventional energy. Also solar nanophotocatalytic degradation seems to be the most appealing choice for the complete degradation and substantial mineralization of alizarin red S and may be extended to treat real industrial wastewater.

\section{Acknowledgement}

The support of the Principal, J.B. College of Science, Wardha is greatly acknowledged.

\section{References}

[1] T.E. Agustina, H.M. Ang, V.K. Vareek, A review of synergistic effect of photocatalysis and ozonation on wastewater treatment, J. Photochem. Photobiol. C Photochem. Rev. 6(4) (2005) 264-269.

[2] B. Neppolian, H.C. Choi, S. Sakthivel, B. Arabindoo, V. Murugesan, Solar/UVinduced photocatalytic degradation of three commercial textile dyes, Jour. Hazard. Mater. 89(2-3) (2002) 303-317.

[3] N. Danesvar, D. Salari, A.R .Khataee, Photocatalytic degradation of azo dye acid red 14 in water: investigation of the effect of operational parameters, J. Photochem. Photobiol. A 157(1) (2003) 111-116.

[4] U.I. Gaya, A.H. Abdullah, Heterogeneous photocatalytic degradation of organic contaminants over titaniumdioxide: A review of fundamental, progress and problems, J. Photochem. Photobiol. C: Photochem. Rev. 9 (2008) 1-12.

[5] M.A. Rauf, S.S. Ashraf, Fundamental principles and application of heterogeneous photocatalytic degradation of dyes in solution, Chem. Eng. J. 151 (2009)10-18.

[6] N. Hadj Salah, M. Bouhelassa, S. Bekkouche, A. Boultif, Study of photocatalytic degradation of phenol, Desalination 166 (2004) 347-354.

[7] T.Y. Zhang, L.Y. You, Y.L. Zhang, Photocatalytic reduction of pchloronitrobenzeneon illuminated nano-titanium dioxide particles, Dyes Pigments 68 (2006) 95-100.

[8] N.P. Ndasi, M. Augustin, T.J. Bosco, Biodecolourisation of textile dyes by local microbial consortia isolated from dye polluted soils in ngaoundere. Int. J. Environ. Sci. 1(7) (2011) 1403-1419.

[9] S. Anandan, A. Vinu, K.L.P. Sheeja Lovely, N. Gokulakrishnan, P. Srinivasu, T. Mori, V. Murugesan, Sivamurugan, K. Ariga, Photocatalytic activity of Ladoped $\mathrm{ZnO}$ for the degradation of monocrotophos in aqueous suspension, J. Mol. Catal. A: Chem. 266 (2007) 149-157.

[10] O.B. Akpor, M. Muchie Remediation of heavy metals in drinking water and wastewater treatment systems: processes and applications, Int. Jour. Phy. Sci. 5(12) (2010) 1807-1817.

[11] S. Asad, M.A. Amoozegar, A.A. Pourbabaee, M.N. Sarbolouki, S.M. Dastghei, Decolorization of textile azo dyes by newly isolated halophilic and halotolerant bacteria, Bioresour. Technol. 98(11) (2007) 2082-2088.

[12] C.S. Turchi, D.F. Ollis, Photocatalytic degradation of organic water contaminants: Mechanisms involving hydroxyl radical attack, Jour. Catal. 122(1) (1990)178-192.

[13] M. Anpo, T. Shima, S. Kodama, Y. Kubokawa, Photocatalytic hydrogenation of propyne with water on small particle titania: size quantization effects and reaction intermediates, J. Phy. Chem. 91(16) (1987) 4305-4310.

[14] D. Li, H. Haneda, S. Hishita, N. Ohashi, Visible-light driven N-F co-doped $\mathrm{TiO}_{2}$ Photocatalysts, Optical characterization, photocatalysis and potential application to air purification, Chem. Mater. 17(10) (2005) 2596-2602.

[15] C. Lizama, J. Freer, J. Baeza, H. Mansilla, Optimsed photodegradation of Reactive Blue 19 on $\mathrm{TiO}_{2}$ and $\mathrm{Zn}$ suspensions, Catal. Today 76 (2002) 235-246.

[16] A. Zuorro, R. Lavecchia, Evaluation of $\mathrm{UV} / \mathrm{H}_{2} \mathrm{O}_{2}$ advanced oxidation process (AOP) for the degradation of diazo dye Reactive Green 19 in aqueous solution, J. Desal. Water TreaT. 52(5-7) (2014) 1571-1577.

[17] H. Lin, C.P. Huang, W. Li, C. Ni, S.I. Shah, Y.H. Tseng, Size dependency of nanocrystalline $\mathrm{TiO}_{2}$ on its optical property and photo-catalytic reactivity exemplified by 2-chlorophenol, Appl. Catal. B Environ. 68(1-2) (2006) 1-11.

[18] L.K. Adams, D.Y. Lyon, P.J.J. Alvarez, Comparative eco-toxicity of nanoscale $\mathrm{TiO}_{2}$, $\mathrm{SiO}_{2}$, and $\mathrm{ZnO}$ water suspensions, Water Res. 40(19) (2005) 3527-3532.

[19] N.P. Mohabansi, V.B. Patil, M.K.N. Yenkie, A comparative study on photo degradation of methylene blue dye effluent by advanced oxidation process by using $\mathrm{TiO}_{2} / \mathrm{ZnO}$ photo catalyst, Rasayan J. Chem. 4(4) (2011) 814-819. 
[20] A. Fujishima, T.N. Rao, D.A. Tryk, Titanium dioxide photocatalysis, J. Photochem. Photobiol. C Photochem. Rev. 1 (2000) 1-21.

[21] D.S. Kim, S.Y. Kwak, The hydrothermal synthesis of mesoporous $\mathrm{TiO}_{2}$ with high crystallinity, thermal stability, large surface area, and enhanced photocatalytic activity, Appl. Catal. A Gen. 323 (2007) 110-118.

[22] H. Fan, T. Jiang, L. Wang, D. Wang, H. Li, P. Wang, et al., Effect of $\mathrm{BiVO}_{4}$ crystalline phases on the photo induced carriers behavior and photocatalytic activity, Jour. Phy. Chem. 116(3) (2012) 2425-2430.

[23] D.S. Kim, S.Y. Kwak, The hydrothermal synthesis of mesoporous $\mathrm{TiO}_{2}$ with high crystallinity, thermal stability, large surface area, and enhanced photocatalytic activity, Appl. Catal. A Gen. 323 (2007) 110-118.

[24] O. Zucas, H. Budiman, N. Hamim, Synthesis of $\mathrm{ZnO}$ nanoparticles for microwave-induced rapid catalytic decolorisation of congo red dye, Adv. Mater. Lett. 4(9) (2013) 662-667.

[25] H. He, S. Yang, K. Yu, Y. Ju, C. Sun, L. Wang, Microwave induced catalytic degradation of crystal violet in nano-nickel dioxide suspensions, J. Hazard Mater. 173 (2012) 393-400.

[26] G. Lofrano, L. Rizzo, M. Grassi, V. Belgiorno, Advanced oxidation of catechol: A comparison among photocatalysis, fenton and photo-fenton processes, Desalination 249 (2009) 878-883.

[27] U.G. Akpan, B.H. Hameed, Parameters affecting the photocatalytic degradation of dyes using $\mathrm{TiO}_{2}$-based photocatalysts: A review, J. Hazard. Mater. $170(2-3)$ (2009) 520-529.
[28] S. Ravichandran, K. Ramanathan, Molecular interactions with polyacrylamide in ethanolamine, diethanolamine, and triethanolamine solutions measured ultrasonically, Polymerplast. Technol. Eng. 47 (2008) 169-173.

[29] R. Kuppa, V.S. Moholkar, Physical features of ultrasound-enhanced heterogeneous permanganate oxidation, Ultrason. Sonochem. 17 (2010) 123131.

[30] Y.L. Pang, A.Z. Abdullah, S. Bhatia, Review on sonochemical methods in the presence of catalysts and chemical additives for treatment of organic pollutants in wastewater, Desalination 277 (2011)1-14.

[31] S. Vajnhandl, A.M.L. Marechal, Ultrasound in textile dyeing and the decolourization /mineralization of textile dyes, Dyes Pigments 65 (2005) 89101.

[32] S. Thirumaran, R. Murugan, N. Prakash, Acoustic study of intermolecular interactions in binary liquid mixture, J. Chem. Pharm. Res. 2(1) (2010) 53-61.

[33] E. Zorebski, M. Geppert-Rybczynska, B. Maciej, Densities, speeds of sound, and isentropic compressibilities for binary mixtures of 2-ethyl-1-hexanol with 1pentanol, 1-heptanol, or 1-nonanol at the temperature $298.15 \mathrm{~K}$, J. Chem. Eng. Data 55(2) (2010) 1025-1029.

[34] M.V. Rathnam, K. Jain, S. Mankumare, M.S. Kumar, Viscosity, density, and refractive index of some (ester + hydrocarbon) binary mixtures at $303.15 \mathrm{~K}$ and 313.15 K, J. Chem. Eng. Data. 50(2) (2005) 325-329.

[35] R.R. Naik, S.V. Bawankar, Ultrasonic study of pyridoxine solutions at different temperatures and concentrations, Russian J. Chem. A 89 (2015) 152-158. 